\title{
Application of Biological Analysis in Life Science and Medicine Research on Biology Analysis and Detection
}

\author{
Xie GenTan ${ }^{1}$, Lin $\mathrm{Na}^{2}$, Bi ZhiLi ${ }^{3}$, Shan Jie ${ }^{4}$, Li HongMei ${ }^{5}$ \\ Binzhou Polytechnic, Binzhou, Shandong 256600, China \\ Email:121258618518@qq.com, ${ }^{2}$ bz3383102@163.com, ${ }^{3}$ beelili@126.com
}

\begin{abstract}
The generation, development, and applications of modern organic analysis was introduced. Emphases are put on two parts: applications of modern organic and biological analysis to life science and pharmaceutical analysis; applications of modern organic and biological analysis to organic analytical sepa- rat.
\end{abstract}

Keywords: modern organic analysis, biological analysis, application

\section{INTRODUCTION}

Modern organic analysis is a new and popular frontier subject of organic chemistry and analytical chemistry. It is an important way for people to know and understand the organic world. With the emergence of various new methods, technologies, theories and instruments for organic and biological analysis, modern organic and biological analysis has been widely used in the field of scientific research Computer science is a new and popular frontier subject which has a very large development space and keeps pace with the times. Therefore, modern organic and biological analysis has broad application prospects in the fields of industry, agriculture, national defense construction, medicine and so on[1]. At the same time, modern organic and biological analysis plays an important role in the development and establishment of many new disciplines such as life science, materials science, environmental science, resources and energy science, information science and so on.

\subsection{Related Work}

Application of modern organic and biological analysis in life science and pharmaceutical analysis. Direct determination of alliin in garlic by 1.1 electrospray ionization mass spectrometry.Modern medical research has confirmed that alliin in garlic has the effects of lowering blood fat, improving body immunity, sterilization, bacteriostasis, anti-cold, anti-aging, promoting blood circulation, preventing cancer and anti-cancer. In recent years, the off-line detection of alliin in garlic has been realized by HPLC or LC-MS. however, these methods require tedious sample pretreatment (such as inactivation, separation, extraction, preconcentration of alliin hydrolase, etc.) and cannot directly analyze alliin in garlic tissue. A new atmospheric ionization technique has been widely used in the analysis of complex matrix samples[2]. However, the ionization technology which can directly obtain the deep material information in the sample will further expand the application scope of atmospheric ionization technology. Therefore, the development of a method for the direct analysis of alliin in garlic cells without sample pretreatment is of great significance for the analysis of unstable active molecules in biological tissues. Zhang Hua et all of East China University of Science and Technology used electrospray ionization mass spectrometry (iEESI-MS) to detect garlic Allium in garlic tissues without direct sample pretreatment. The results of direct ieesi-ms analysis of garlic tissue showed that ieesi was a soft ionization technology, which could obtain the signal of deep substances in the tissue[3]. It had important reference significance for the analysis of unstable active molecules in biological tissue.Spectroscopic study on the interaction between fluoroporphyrin anthraquinone compounds and DNA Porphyrins are good macrocyclic aromatic photosensitizers because of their strong light absorption and luminescence properties. Anthraquinones have good characteristics of DNA Photofragmentation. They are easy to insert into DNA base pairs and break DNA by different mechanisms. Fluorinated compounds have strong physiological activities, which may interact with biological tissues to show anti-tumor effect. Zhao Shengfang et al[4]. Of Huanggang Normal University synthesized two peptide bonded fluoroporphyrin anthraquinone compounds and their metal zinc complexes under microwave irradiation. The interaction between the two synthesized target compounds and plasmid DNA was investigated by UV Vis spectroscopy and fluorescence spectrometric titration, and the interaction mode between them and DNA was discussed. That is, porphyrin dipeptide anthraquinone compounds and DNA self stacking external bonding[5]. This research will be widely 
used in life science, medicine and coordination chemistry.

\subsection{Our Contribution}

Detection of nucleic acids based on DNA self-assembly enzyme-free cyclic amplification quenching chemiluminescence[6]. Quadruplex DNase is a kind of nucleic acid molecule with catalytic function. Because the G-rich nucleic acid molecule can form quadruplex structure in the presence of heme, it shows activity similar to horseradish peroxidase, and can catalyze $\mathrm{H} 2 \mathrm{O} 2$ oxidation of luminol to produce chemiluminescence[7]. Based on the goal of catalyzing DNA self-assembly, Chu Zhidan et al. From Guangxi Normal University, based on the fact that $G$ quadruplex is a special DNA secondary structure formed by guanine rich DNA sequence, it can show strong peroxidase catalytic activity when combined with heme, A novel chemiluminescence biosensor system without enzyme cycle amplification quenching was constructed for DNA detection. The sensor system is simple, low consumption and sensitive. This research will be widely used in life science, medicine and biology.Detection of adenosine by chemiluminescence catalyzed by G-quadruplex DNAzyme

Adenosine plays an important role in the regulation of physiological activity of various biological tissues and organs. Therefore, it is of great significance to detect adenosine content in organisms. Chemiluminescence detection has the advantages of high sensitivity, wide linear range and fast analysis speed. Li Mei et al. Of Guangxi Normal University used DNA rich in G base to form $g$ quadruplex structure in the presence of heme and $\mathrm{K}+$, and showed the characteristics of peroxidase activity, A novel method for the detection of adenosine based on DNA strand replacement reaction and endonuclease assisted signal amplification was developed. The detection limit is $0.5^{\wedge} \mathrm{mol} / \mathrm{L}$. the method can be used for the determination of adenosine in human serum[8]. Secondary metabolites of mangrove fungus Fusarium sp. 301 from the South China Sea.Mangrove ecosystem is a kind of woody plant community distributed in tropical and subtropical intertidal zone, which has the unique forest type of marine environment. The mangrove endophytic fungi bred in its special environment are the main degradation of this ecosystem, and also the second largest ecological community of marine fungi. Since 1994, studies on the secondary metabolites of marine fungi at home and abroad have shown that mangrove endophytic fungi contain abundant compounds with novel structures and good pharmacological activities in anti-tumor, anti-oxidation, antifungal, bacterial and other aspects. The active metabolites of marine fungi have become one of the important sources of new drugs. In order to find the secondary metabolites of marine fungi with novel structure and pharmacological activity, Fang Ping, et al., Zhongshan University, studied the secondary metabolites of an endophytic fungus Fusarium sp. 301 from mangrove mangrove, Haikou. The secondary metabolites were isolated and purified according to their physicochemical properties, reverse phase silica gel, gel column chromatography and high performance liquid chromatography. Five streptomycetin compounds were isolated and identified by spectral analysis and literature data comparison. This research will be widely used in life science, biology and medicine[9].

Electrochemical oxidation of ambroxol hydrochloride.Ambroxol hydrochloride (AMB), trans-4 - [(2-amino-3,5-dibromobenzyl) amino] cyclohexanol hydrochloride, is a specific drug for the treatment of respiratory diseases. It has different scavenging abilities to reactive free radicals (ROS), hydroxyl radicals (Ho ·) and superoxide anion (. It has little or no effect on hydrogen peroxide ( $\mathrm{H} 2 \mathrm{O} 2)$. Therefore, it is of great significance to study the oxidation process of AMB. Therefore, sun jiejuan et al. [9] from Northwestern University studied the voltammetric behavior of ambroxol hydrochloride (AMB) in acidic aqueous solution, and explained the oxidation mechanism of AMB with reference to the voltammetric characteristics of substituted aniline. This research will be applied in the research of life science and medicine.

\section{BACKGROUND}

\subsection{Probabilistic Automata}

Application of modern organic and biological analysis in the science of organic analysis and separation.Chitosan derived calix [4] arene bonded silica gel has strong chemical reaction ability and biocompatibility for the separation and analysis of eight monosubstituted benzenes; The pore size of calixarene can be adjusted, and the conformation and substituents can be controlled artificially[10]. Lu Jing et al. Of Zhengzhou University combined the advantages of the two to prepare a new type of drug. Eight kinds of benzene were separated and analyzed by the fixed phase. Their method is to study the separation, thermodynamics and hydrophobicity of eight kinds of single substituted benzene (aniline, benzaldehyde, phenol, toluene, chlorobenzene, bromber benzene, iodobenzene and butylene) by using the self-made chitosan derivative calix arene bonded silica gel stationary phase (CBS4). The results showed that the separation time of 8 kinds of single substituted benzene was short and the separation effect was good, which was in line with the mechanism of reversed phase chromatography; The retention time 
of analytes decreases with the increase of column temperature; Hydrophobic action plays an important role in the separation of single substituted benzene[10]. The research will be applied in the analysis and separation science, environmental science and biology.

Spectral characteristics of $\mathrm{cu} 2+$ carboxymethacetan complex and its application as a phlegm regulator, which is used to treat the phlegm viscosity caused by bronchitis, asthma and other diseases, and cough up difficult, but the excessive dosage has medical side effects. Therefore, Lin $\mathrm{Yu}$ et al of Guangxi University, based on the molecular structure characteristics of carboxymethastin, speculates that the molecule should have the condition of complexation with metal ions. The possibility of complexation of carboxymethactam with various metal ions was investigated by UV visible spectrophotometry. Their research found that carbendan and $\mathrm{cu} 2+\mathrm{can}$ form stable complex, which has a maximum characteristic absorption peak at $237 \mathrm{~nm}$. The complex ratio of carbomestane cu2+ was determined to be.The stability constant of the complex is $4.98 \times 109$. The maximum molar absorption coefficient of the complex at $237 \mathrm{~nm}$ is $4.74 \times$ 1031/ (mol • $\mathrm{cm}$ ). A new UV spectrophotometry method based on cu2+ complexation for the quantitative determination of carboxymethactam is established. The method is simple, rapid, sensitive and accurate, and has been successfully applied to the analysis of pharmaceutical products. The results are in line with scalar.

\section{CONCLUSION}

The conclusion is a new method, new technology and instrument with organic analysis and biological analysis.With the continuous development of new theories, modern organic analysis and biological analysis have developed rapidly, and provide a strong guarantee for human production, life, survival and sustainable development.[10] The establishment and development of modern organic and biological analysis has promoted the formation and development of life science, environmental science, resources and energy science and material science, and has shown a broad application prospect in the fields of industry, agriculture, national defense, aerospace, communication science, information science, Medical Pharmacy and so on. With the deepening of modern organic and biological analysis, modern organic and biological analysis is developing in the direction of accuracy, sensitivity, novelty, online, trace trace quantification and rapid development. The future of modern organic and biological analysis will be brighter and more brilliant. Fried thinks the roadmap is bold, but the latter two themes are not easy to do [11].Various NH funds and tutor awards will attract more clinical researchers, but the specific effect remains to be seen. The concept of "chief researcher" in $\mathrm{NH}$ roadmap should be redefined. In the new model of the roadmap, researchers are no longer isolated "islands", and each member will play an important role and play an equal role in the whole plan. Therefore, each member of the team should be given appropriate resources and enough rewards, which is particularly important for senior members with low seniority. In a word, the roadmap needs the joint efforts of all research institutes and researchers. $\mathrm{NH}$ roadmap project is another large-scale biomedical research plan after the human genome project. It advocates crossover, encourages risk-taking, promotes quantitative and data sharing, promotes large-scale biological research, and strives to promote the transformation of basic research into clinical results. Its implementation will have a great impact on future biomedical research.

\section{REFERENCES}

[1] Chen Huanwen , Yang Shuiping , Li Ming , et al. Sensitive detection of native proteins using extractive electrospray $i \neg$ onization mass spectrometry $[\mathrm{J}]$. Angewandte Chemie International Edition, 2010 , 49 , 3053-3056.

[2] Chen Huanwen, Gamez G, Zenobi R. What can we learnfrom ambient ionization techniques[J]. Journal of the A $\neg$ merican Society for Mass Spectrometry, 2009, 20: $1947 \neg$ one thousand nine hundred and sixty-three.

[3] Zhang Hua, Zhu Liang, Wang Nannan, et al. Direct detection of alliin C / garlic in garlic by internal spray ionization mass spectrometry. Proceedings of the Seventeenth National Symposium on organic and biological analysis. Nanning: Guangxi University, 2013.

[4] Zhao Shengfang, chennianyou, shenhuihui, et al. Spectroscopic study of porphyrin anthraquinone [C] Proceedings of the 17th National Symposium on organic and biological analysis. Nanning: Guangxi University, 2013.

[5] Chu Zhidan, Huang Yong, Li Mei, et al. Self assembly of DNA based enzyme free circulating release Papers of the 17th National Symposium on organic and biological analysis. Nanning: Guangxi University, 2013.

[6] Li Mei, Huang Yong, Chu Zhidan, et al. Based on DNA chain replacement enzyme assisted signal Amplify the catalytic chemilu minescence detection of adenosine c] / Proceedings of the 17th National Symposium on organic and biological analysis. Nanning: Guangxi University, 2013. 
[7] Gopl B, Chauhan M. Diversity and conservation in the Sundarban mangrove ecosystem [J]. Aguatic Science, 2006, 68: 338-354.

[8] Fang Ping, Li Jing, Liu Lan. The secondary metabolites of Fusarium sp. 301 of mangrove fungi in the South China Sea c] / / Proceedings of the 17th National Symposium on organic and biological analysis. Nanning: Guangxi University, 2013.

[9]Sun jiejuan, Wuyi, songjunfeng. Electrochemical oxidation of ambroxol hydrochloride[C] / / the proceedings of the 17th National Symposium on organic and biological analysis. Nanning: Guangxi University, 2013.

[10] Lu Jing, tangchao, Liupu, et al. Chitosan derivative calix [4] aromatics bonded silicon Separation and analysis of eight kinds of benzene with fixed rubber c] Proceedings of the 17th National Symposium on organic and biological analysis. Nanning: Guangxi University, 2013.

[11] Lin Yu, weixiaoling, Gong Qi, et al. Spectral characteristics and application of cu2+ carboxystan complex c] / / Proceedings of the 17th National Symposium on organic and biological analysis. Nanning: Guangxi University, 2013.

[12] P. Xu, N. Na, A. M. Mohamad, Investigation the application of pristine graphdiyne (GDY) and boron-doped graphdiyne (BGDY) as an electronic sensor for detection of anticancer drug, Computational and Theoretical Chemistry, 1190(2020): 112996.

[13] Z. Li, X. Tian,Effects of Divalent Calcium Ion on the Flame Retardancy and Pyrolysis Products of Synthetic Polyvinyl Alcohol, Journal of Applied Science and Engineering Innovation,7(2020)125-131.

[14] P. Xu, N. Na, Study on Antibacterial Properties of Cellulose Acetate Seawater Desalination Reverse-Osmosis Membrane with Graphene Oxide, Journal of Coastal Research, 105(2020)246-251. 